\title{
Relationship between the dietary intake of water and skin hydration
}

\author{
Relação entre a ingestão dietária de água e a hidratação cutânea
}

\author{
Maria Lídia Palma ${ }^{1}$, Carla Monteiro ${ }^{1}$, Liliana Tavares ${ }^{1}$ Maria Julia Bujan $^{2}$ \& Luís Monteiro Rodrigues ${ }^{1,3}$ \\ CBIOS - Universidade Lusófona's Research Center for Health Science and Technologies (UDE), \\ Campo Grande, 376, 1749-024, Lisboa, Portugal \\ ${ }^{2}$ Universitad de Alcalá Henares, Faculty of Medicine, Madrid, Spain \\ ${ }^{3}$ Universidade de Lisboa (Faculty of Pharmacy)- Dep. Pharmacological Sciences \\ Email: p1814@ulusofona.pt (Lidia Palma)
}

\begin{abstract}
Several studies about the importance of water in the hydration of the human skin suggest that higher water intake might have a positive impact on the skin. When measuring water consumption, it must be taken into account that the water comes from various sources. With this in mind, we evaluated the impact of an increased inflow of $2 \mathrm{~L}$ of water per day, on two groups with different daily water intakes. Results suggest that there is an improvement in the epidermal and dermal hydration obtained by standard methods. The magnitude of this impact on skin hydration is greater in the group that regularly consumes less water. This suggests that an increase in water consumption has more beneficial effects on the skin health of those individuals that consume less water per day. These data are consistent with the published literature, which therefore justifies further research into this topic.
\end{abstract}

Keywords: Skin Hydration; dietary water; QFA, nutrients.

\begin{abstract}
Resumo
Os vários estudos sobre a importância da água na hidratação da pele humana vêm sugerindo que o aumento da ingestão de água se revela positivamente na pele. Considerando que a contabilização da água consumida deve atender às suas várias origens, avaliámos em dois grupos com diferentes consumos de água total, o impacto do aumento do aporte de $2 \mathrm{~L} /$ dia de água bebida. Os resultados sugerem que, existe uma melhoria da hidratação epidérmica e dérmica obtida por métodos de referência. A magnitude do impacto sobre a hidratação cutânea é maior no grupo que regularmente consome menos água, sugerindo que o aumento do consumo de água tem mais efeitos benéficos na saúde cutânea dos indivíduos que consomem menos água por dia. Estes dados estão de acordo, com a literatura publicada, justificando o interesse no aprofundamento do tema.
\end{abstract}

Palavras-chave: Hidratação cutânea; Água da dieta; QFA, nutrientes. 


\section{Introduction}

We know that our metabolic pool of water which is essential to human physiology, results from water present in food, water produced by metabolic pathways and water we drink ${ }^{[1]}$. However, unlike other essential nutrients, there is no clear definition for our daily water needs. This might be partly explained by the set of highly sensitive neurophysiological adaptations and adjustments that occur for a wide range of ingested fluids, in order to maintain the body's osmolality and hydration. However, it is also accepted that it is difficult to define an ideal water intake value to ensure an adequate "healthy" hydration for all people in all environmental conditions. Different levels of physical activity and metabolism are obvious determinants of this condition. The characterization study of the pattern of water consumption carried out in the Portuguese population in $2010^{[2]}$, allowed researchers to identify the contribution of various sources to the total amount of water consumed. A survey instrument to measure food frequency (FFQ) was used to measure the total water intake of the study population as the sum of drunk water $(\mathrm{AgB})$, water from food $(\mathrm{AgD})$ and metabolic water, and to identify the respective contributions.

Once the pattern of water consumption has been established, the impact on the physiology of the skin can be assessed. This is particularly significant if we keep in mind the importance of water on the skin's cellular dynamics $^{[3]}$ and the composition of $\mathrm{NMF}^{[4]}$ (Natural Moisturizing Factor), an important component of epidermal hydration that also has an impact on the skin's appearance $^{[5]}$.

The objective of the present study is to evaluate the impact of water intake on in vivo skin hydration in a group of young, healthy women.

\section{Material and methods}

A sample of 11 healthy, female volunteers was selected. They were not taking any systemic or topical medication, had no smoking habits or any type of addiction, were aged between 22 and 34 (mean $24.5 \pm$ 4.27)years old, and were willing to participate. All participants were informed about the objectives and procedures of the study. After expressing their availability to participate, they completed the informed consent and agreed to comply with the study's methodology. The study protocol was approved by the

\section{Introdução}

Sabemos que a nossa pool metabólica de água, essencial em toda a fisiologia humana, provêm da água presente nos alimentos, da água produzida por via metabólica e da água ingerida ${ }^{[1]}$. Contudo, ao contrário do que acontece com outros nutrientes fundamentais, não existe para a água uma definição clara sobre as necessidades diárias, facto que pode ser parcialmente explicado pelo conjunto altamente sensível de adaptações neurofisiológicas e ajustes que se fazem sentir para um vasto leque de quantidades ingeridas de líquidos, de modo a manter a osmolaridade e a hidratação do organismo. Mas admite-se também, não ser possível definir um valor único de ingestão de água, que assegure uma hidratação adequada para a saúde de todas as pessoas aparentemente saudáveis em todas as condições ambientais, já que, os diferentes niveis de actividade física e de metabolismo, são determinantes óbvios. O estudo de caracterização do padrão do consumo de água levado a cabo na população portuguesa em $2010^{[2]}$, permitiu identificar o contributo dos vários aportes na quantidade total de água consumida, utilizando como instrumento de medida um inquérito de frequência alimentar (QFA), através do qual foi possível contabilizar o aporte total de água da população em estudo como o somatório da água bebida $(\mathrm{AgB})$, água da dieta $(\mathrm{AgD})$ e água metabólica e, o contributo das várias componentes no aporte total.

Uma vez estabelecido o padrão de consumo de água podemos avaliar a importância que este factor tem sobre a fisiologia da pele em especial, tendo presente a importância que a água tem sobre a dinâmica ${ }^{[3]}$ celular da pele e na composição do $\mathrm{FHN}^{[4]}$ (Factor Hidratação Natural) componente importante da hidratação epidérmica, com impacto na aparência da pele $\mathrm{e}^{[5]}$.

O presente estudo tem como objectivo principal avaliar o impacto do aporte dietário de água na hidratação da pele in vivo, num grupo de mulheres jovens, saudáveis.

\section{Material e métodos}

Foi seleccionada uma amostra de 11 voluntárias do género feminino saudáveis, sem medicação sistémica ou tópica, sem hábitos tabágicos ou qualquer tipo de adicção com idades compreendidas entre os 22 e os 34 (média 24,5 $\pm 4,27$ ) anos, que revelaram disponibilidade e interesse em participar. Todos as participantes foram esclarecidas quanto aos objectivos e procedimentos inerentes ao estudo e, após expressarem a sua vontade em participar, preencheram o consentimento informado e acederam em cumprir a metodologia do estudo. $\mathrm{O}$ 
Institutional Ethics Committee and conducted according to the principles outlined in the Declaration of Helsinki and respective amendments ${ }^{[6]}$.

We also assessed the volunteer's dietary habits concerning water consumption using the food frequency questionnaire (FFQ) and the water consumption survey. The FFQ used is a self-assessment questionnaire, developed by the Hygiene and Epidemiology Department (FMUP) and based on the model proposed by Willett ${ }^{[7]}$. The Food Processor Plus ${ }^{\circledR}$, version 5.0 (ESHA Research, USA) software was used to convert food into nutrients. This software is based on the U.S. Department of Agriculture food composition table, and includes raw and / processed food. Moreover, we have added the nutritional content of food and typical portuguese cuisine to this database. This was done according to information from the national food composition table and other studies that assessed Portuguese food composition as described in detail by other authors.

Since the total water associated with the feeding function results from the sum of water ingested as a liquid and as a dietary component of food ${ }^{[8]}$, volunteers participating in the study were selected according to the total water consumption. This included (a) the consumed water (as water, tea and infusions consumed between meals), (b) the diet water measured by the FFQ which included the water from five food groups - Coca Cola, Pepsi Cola or other; Ice tea, other soft drinks, fruit juices or packed nectars; milk, vegetable soup and broth, and (c) the metabolized water ${ }^{[9]}$ through the macronutrients oxidative pathway (fat, carbohydrate and protein).

Two groups were considered based on the analysis of total water content in the diet - group $1(n=5)$ with an average water consumption of $1700.4 \pm 323 \mathrm{ml} /$ day and group $2(\mathrm{n}=6)$ with a average water consumption of $3420.4 \pm 1114.9 \mathrm{ml} /$ day (Table 1). protocolo de estudo foi aprovado pela comissão de ética institucional e conduzido de acordo com a Declaração de Helsínquia e respectivas alterações ${ }^{[6]}$.

Foram ainda considerados os hábitos dietários de consumo de água dos voluntários através de questionário de frequência alimentar (QFA) e inquérito de consumo diário de água. O QFA utilizado, de administração directa, preenchido pelos voluntários, foi desenvolvido no Serviço de Higiene e Epidemiologia (FMUP), tendo por base o modelo proposto por Willett $^{[7]}$. Para a conversão dos alimentos em nutrientes foi utilizado o programa informático Food Processor Plus ${ }^{\circledR}$, versão 5.0 (ESHA Research, USA), que utiliza a tabela de composição de alimentos do Departamento de Agricultura dos EUA e inclui alimentos crus e/ou processados. Para além disso, foram acrescentados a esta base de dados, os conteúdos nutricionais de alimentos ou pratos culinários tipicamente portugueses, de acordo com informações nacionais da tabela de composição dos alimentos portugueses e de outros estudos que avaliaram a composição de alimentos portugueses, como descrito em detalhe por outros autores.

Atendendo a que a água total associada a nossa função alimentar resulta do somatório da água ingerida como líquido e como componente (dietária) dos alimentos ${ }^{[8]}$, as voluntárias que participaram no estudo foram seleccionadas de acordo com o consumo total de água que incluiu (a) a água consumida (água chás e infusões consumidas fora das refeições), (b) a água da dieta contabilizada via QFA que incluiu o conteúdo em água de cinco grupos de alimentos - coca-cola, pepsi-cola ou outras; Ice tea; outros refrigerantes, sumos de fruta ou néctares embalados; leite; sopa de legumes e canja, e (c) a água ${ }^{[9]}$ metabolizada através da via oxidativa dos macronutrientes (lípidos, carbohidratos e proteína).

Com base na análise dos conteúdos em água total das dietas foram considerados dois grupos; o grupo $1(\mathrm{n}=5)$, com um consumo médio de água de $1700,4 \pm 323 \mathrm{ml} / \mathrm{dia}$ e o grupo $2(\mathrm{n}=6)$, com um consumo médio de água de $3420,4 \pm 1114,9 \mathrm{ml} /$ dia (tabela 1).

Table 1- Descriptive analysis of the Total Water consumption of the studied population Tabela 1-Análise descritiva do consumo de Água Total da população estudada

\begin{tabular}{|l|c|c|c|c|c|}
\cline { 2 - 6 } \multicolumn{1}{c|}{} & $\mathrm{n}$ & $\begin{array}{c}\text { Minimum } \\
\text { Minimo }\end{array}$ & $\begin{array}{c}\text { Maximum } \\
\text { Máximo }\end{array}$ & $\begin{array}{c}\text { Mean } \\
\text { Média }\end{array}$ & $\begin{array}{c}\text { Std Deviation } \\
\text { Desvio Padrão }\end{array}$ \\
\hline Group / Grupo 1 & 5 & 1211.10 & 1998.10 & 1700.4 & 325.5 \\
\hline Group / Grupo 2 & 6 & 2264.7 & 4956.1 & 3420.4 & 1141.9 \\
\hline
\end{tabular}


The study lasted for 4 weeks, with assessments at day zero (T0), day fifteen (T1) and day thirty (T2). During the study the volunteers accepted to maintain their dietary patterns and habits of topical administration of toiletries and body care in anatomical locations under study (leg, forearm, hand, forehead and zygomatic area). However, the application of these products was restricted to no later than $24 \mathrm{~h}$ before measurements. The dietary reference value (DVR - Dietary Reference Values) for women stated by the European Food Safety Authority $\left(\mathrm{EFSA}^{\left[{ }^{10]}\right.}\right.$ is $2 \mathrm{~L} /$ dia. Bearing this in mind, over a period of one month we added $(2000 \mathrm{ml})$ of bottled water (mineral water Vitalis Unicer ${ }^{\circledR}$ SA) to the normal dietary regimen of each volunteer. Table 2 shows the composition of this drinking water standard.
O estudo teve a duração de 4 semanas, com avaliações no dia zero (T0), no dia quinze (T1) e no dia 30 (T2). Durante o estudo as voluntárias comprometeram-se a manter o seu padrão alimentar e os seus hábitos de administração tópica de produtos de higiene e cuidados corporais nas zonas anatómicas em estudo (perna, antebraço, mão, zona zigomática e fronte) incluindo a restrição de aplicação de produtos de higiene e cuidados corporais nos dias das medições. Nestas condições, considerando, que o valor dietário de referência, (DVR Dietary Reference Values) para as mulheres, da European Food Safety Authority (EFSA) ${ }^{[10]}$ é de 2L/dia, acrescentámos durante um mês, ao normal regime dietário de cada voluntária, 2L $(2000 \mathrm{ml})$ de água engarrafada (água mineral Vitalis ${ }^{\circledR}$ da Unicer S.A.). A Tabela 2 apresenta a composição padrão da água consumida.

Table 2- Description of mineral water used in the study

Tabela 2- Descrição da água mineral utilizada no estudo estudada

\begin{tabular}{l|c}
\hline \multicolumn{1}{c|}{$\begin{array}{c}\text { Mineralization total: 26mg / } \\
\text { Mineralização total 26mg/I }\end{array}$} \\
\hline Silica & $10 \mathrm{mg} / \mathrm{l:}$ \\
Bicarbonate / Bicabornato & $<1.0 \mathrm{mg} / 1$ \\
Chloride / Cloreto & $7.0 \mathrm{mg} / 1$ \\
Nitrate / Nitrato & $1.2 \mathrm{mg} / 1$ \\
Sodium / Sódio & $3.8 \mathrm{mg} / 1$ \\
Calcium / Cálcio & $0.4 \mathrm{mg} / 1$ \\
Magnesium / Magnésio & $0.7 \mathrm{mg} / 1$ \\
Dry residue / Residuo Seco & ------ \\
pH & 4.8 \\
\hline
\end{tabular}

All subjects had a normal Body Mass Index (BMI) according to the WHO criteria $^{[11]}$ (between 19.9 and $24.9 \mathrm{~kg} / \mathrm{m} 2$ ), confirmed by the Quetelet's formula BMI $=$ weight $/$ height $^{2}$.

The water from extra and intracellular compartments was quantified by a bioimpedance method ${ }^{[12]}$ using the Bodystat QuadScan $4000 \AA$, marketed by Bodystat (Isle of Man, UK). This equipment operates on several frequencies. The Total Body Water (TBW Total Body Water) values are calculated at $200 \mathrm{kHz}$ and the Extracellular Water in compartment (ECW Extracellular Water) values are calculated at $5 \mathrm{kHz}$. The values of the Intracellular compartment (ICW Intracellular Water) are obtained indirectly by
Todas as voluntárias apresentaram um Índice de Massa Corporal (IMC) de normal (entre 19,9 and $24,9 \mathrm{Kg} / \mathrm{m}^{2}$ ), confirmado pela formula de Quetelet's- BMI = peso/altura. ${ }^{2}$

A água dos compartimentos extra e intracelular foi quantificada por bioimpedância ${ }^{[12]}$ através co sistema Bodystat ${ }^{\circledR}$ QuadScan 4000, (Bodystat Isle of Man, UK). Os valores de Água Corporal Total (TBW Total Body Water) são calculados a $200 \mathrm{kHz}$ e os de Água no Compartimento Extracelular (ECW Extracellular Water) a $5 \mathrm{kHz}$. Os valores de Água no Compartimento Intracelular (ICW Intracellular Water) são obtidos indirectamente, pela diferença entre os valores obtidos de TBW e ECW. 
calculating the difference between the values of TBW and ECW.

The skin variables considered relevant to the objectives of the study were:

- The epidermal capacitance, measured by the MoistureMeter-SC system ${ }^{\circledR}$ and the MoistureMeter-D (B) (both from Delfin Technology Ltd Kuopio, Finland); these allow for the quantification of superficial and deep water respectively, both expressed in arbitrary units (AU's).

- The trans-epidermal loss of water, (TEWL) evaluated by the Tewameter TM $300 \AA$ system (Courage \& Khazaka, Germany electronics) and considered as an indicator of the cutaneous "barrier" function expressed in g/h.m2.

Prior to measurements volunteers acclimatised for about 15 minutes in the laboratory room environment which was kept under controlled temperature (20-24 ${ }^{\circ}$ C) and humidity (40-60\% RH) conditions.

Analysis of results involved SPSS 19.0, and included a descriptive statistical calculation of uni and varied frequencies, as well as measures of central tendency and dispersion. Hypothesis tests such as the Shapiro - Wilk test to verify the normality of the variables, and the Spearman correlation test, to verify potential correlations between variables, were also applied. A confidence level of $95 \%$ was adopted.

\section{Results}

By adding the same amount of water (2L water) to all volunteers' diets, we aimed to evaluate the impact of this increased intake of water whilst the regular diet habits in both groups were maintained.

No statistically significant differences in BMI, TBW and ICW were found between these two groups, as shown in Table 3. This means that no change in weight and volume, from the water distribution between compartments, resulted from the increased water intake.

Regarding the impact of water intake on skin hydration (Table 4) we find, in group 1, a statistically significant increase in skin hydration in the forehead and forearms in T1 and by the end of the study (T2). In other anatomical areas the statistically significant differences can be observed only at the end of the study. In group 2 the "capacitance" increase can only be observed in the forehead at the end of the study (T2) (Table 4).
As variáveis cutâneas consideradas relevantes para os objectivos do presente estudo foram:

- A capacitância epidérmica avaliada pelo sistema MoistureMeter-SC ${ }^{\circledR}$ (Delfin Tecnology Lda Kuopio, Filand), que permite a quantificação da água superficial, e pelo sistema MoistureMeter-D ${ }^{\circledR}$ (Delfin Tecnology Lda Kuopio, Filand), que permite a quantificação da água profunda, ambas expressas em unidades arbitrárias (UA's).

- A perda trans-epidérmica de água (PTEA) avaliada pelo sistema Tewameter ${ }^{\circledR}$ TM 300 (Courage \& Khazaka, electronics Germany) considerada como um indicador da função de "barreira" cutânea expressa em g/h.m²

Antes da medição foi assegurada a aclimatação das voluntárias ao ambiente do laboratorial, mantendo-se em repouso cerca de 15 minutos em condições de temperatura $\left(20-24^{\circ} \mathrm{C}\right)$ e humidade (humidade relativa 40-60\%) controlada.

Os resultados foram tratados em SPSS 19.0, tendo sido realizada uma estatística descritiva uni variada com cálculo de frequências, medidas de tendência central e de dispersão. Foram ainda aplicados testes de hipóteses de Shapiro - Wilk para verificar a normalidade das variáveis e, a correlação de Spearman com o objectivo de verificar a existência de uma relação estatisticamente significativas com um grau de confiança de $95 \%$.

\section{Resultados}

A adição da mesma quantidade de água (2L de água) a todas as voluntárias pretendeu avaliar o impacto do incremento da ingestão dietária de água, mantendo constantes os hábitos dietários, nos dois grupos.

As variáveis biométricas e o IMC obtidos em ambos os grupos que constam na tabela 3 , demonstram que não existem diferenças estatisticamente significativas no IMC, TBW e ICW nos dois grupos de estudo, o que significa ausência de alterações ponderais e de volume de distribuição de água nos compartimentos do organismo como resultado do aumento dietário de água. Quanto ao impacto do aporte de água na hidratação superficial da pele (tabela 4) verificamos, no grupo 1 , um aumento estatisticamente significativo da hidratação cutânea nas zonas anatómicas da fronte e antebraço, quer em T1, quer no final do estudo (T2). Nas restantes zonas anatómicas o aumento estatisticamente significativos é só observado no final do estudo. No grupo 2 o incremento da "capacitância" epidérmica só é observado na zona anatómica da fronte no final do estudo (T2) (tabela 4). 
Table 3- Biometric variables and body water distribution (check text for abbreviations) Tabela 3- Variáveis biométricas e distribuição de água no organismo (ver significado das abreviaturas no texto)

\begin{tabular}{|l|c|c|c|c|}
\cline { 2 - 5 } \multicolumn{1}{c|}{} & \multicolumn{2}{c|}{ GROUP/ Grupo 1 - n = 5 } & \multicolumn{2}{c|}{ GROUP / Grupo 2 - n = 6 } \\
\cline { 2 - 5 } \multicolumn{1}{c|}{} & T0 & T2 & T0 & T2 \\
\hline $\begin{array}{l}\text { BMI / } \\
\text { IMC }\end{array}$ & $23.22 \pm 1.46$ & $23.24 \pm 1.55(\mathrm{~ns})$ & $21.62 \pm 3.66$ & $21.82 \pm 3.23(\mathrm{~ns})$ \\
\hline TBW & $31.88 \pm 2.43$ & $32.58 \pm 1.09(\mathrm{~ns})$ & $30.33 \pm 2.83$ & $29.75 \pm 1.38(\mathrm{~ns})$ \\
\hline ECW & $14.74 \pm 0.87$ & $15.64 \pm 1.21(\mathrm{~ns})$ & $14.12 \pm 1.09$ & $14.28 \pm 0.84(\mathrm{~ns})$ \\
\hline ICW & $17.00 \pm 1.74$ & $16.3 \pm 1.97(\mathrm{~ns})$ & $15.82 \pm 2.09$ & $15.12 \pm 1.43(\mathrm{~ns})$ \\
\hline
\end{tabular}

Table 4- Superficial hydration (AU's)

Tabela 4- Hidratação superficial (UA's)

\begin{tabular}{|c|c|c|c|c|c|c|}
\hline & T0 & $\mathrm{T} 1$ & $\mathrm{~T} 2$ & $\begin{array}{l}\text { P-Value } \\
\text { (T1 vsT0) }\end{array}$ & $\begin{array}{l}\text { P-Value } \\
\text { (T2 vsT1) }\end{array}$ & $\begin{array}{c}\text { P-Value (T2 } \\
\text { vsT0) }\end{array}$ \\
\hline \multicolumn{7}{|l|}{ Group / Grupo 1 n $=5$} \\
\hline Forehead / Fronte & $32.08 \pm 11.50$ & $53.70 \pm 8.60$ & $66.14 \pm 13.60$ & 0.014 & 0.093 & 0.001 \\
\hline Zygomatic /Zigomática & $24.92 \pm 19.90$ & $25.40 \pm 9.30$ & $47.78 \pm 18.70$ & 0.961 & 0.091 & 0.010 \\
\hline Hand / Mão & $20.60 \pm 11.0$ & $29.00 \pm 9.50$ & $48.80 \pm 7.90$ & 0.105 & 0.023 & 0.020 \\
\hline Forearm /Antebraço & $16.68 \pm 5.70$ & $27.20 \pm 8.10$ & $34.80 \pm 11.10$ & 0.034 & 0.133 & 0.016 \\
\hline Leg / Perna & $18.88 \pm 5.70$ & $29.14 \pm 5.70$ & $40.66 \pm 7.50$ & 0.070 & 0.014 & 0.009 \\
\hline \multicolumn{7}{|l|}{ Group / Grupo 2 n = 6} \\
\hline Forehead / Fronte & $35.78 \pm 5.20$ & $47.90 \pm 15.70$ & $62.45 \pm 9.50$ & 0.147 & 0.064 & 0.002 \\
\hline Zygomatic/Zigomática & $33.32 \pm 20.10$ & $37.32 \pm 18.10$ & $40.57 \pm 12.50$ & 0.712 & 0.347 & 0.429 \\
\hline Hand/ Mão & $22.63 \pm 6.30$ & $31.67 \pm 8.40$ & $36.37 \pm 11.60$ & 0.138 & 0.121 & 0.098 \\
\hline Forearm/Antebraço & $14.20 \pm 5.70$ & $18.67 \pm 4.50$ & $20.73 \pm 4.10$ & 0.162 & 0.199 & 0.052 \\
\hline Leg/ Perna & $19.30 \pm 9.20$ & $28.93 \pm 12.60$ & $28.35 \pm 9.50$ & 0.096 & 0.925 & 0.056 \\
\hline
\end{tabular}

Regarding deep hydration, results are summarized in Table 5. There is a statistically significant increase in deep hydration in group 1 zygomatic area, hand, forearm and leg (Table 5). In group 2, only significant differences can be observed in the anatomic area of the leg and the forehead (Table 5).
O impacto do aporte de água na hidratação profunda, está resumido na tabela 5. A análise dos valores de "capacitância" demonstra que existe um aumento estatisticamente significativo na hidratação profunda no grupo 1 na zona zigomática, mão, antebraço e perna (tabela 5). No grupo 2 só se observam diferenças significativas na zona anatómica da perna e da fronte (tabela 5). 
Table 5- Deep Hydration (AU's)

Tabela 5-Hidratação profunda (UA's)

T0

Group / Grupo 1 n $=\mathbf{5}$

\begin{tabular}{lllllll}
\hline Forehead / Fronte & $31.52 \pm 2.14$ & $32.40 \pm 2.60$ & $33.20 \pm 3.16$ & 0.500 & 0.223 & 0.225 \\
\hline Zygomatic /Zigomática & $32.82 \pm 4.46$ & $31.08 \pm 3.78$ & $34.58 \pm 3.15$ & 0.498 & 0.043 & 0.080 \\
\hline Hand / Mão & $30.72 \pm 9.43$ & $38.58 \pm 4.13$ & $40.18 \pm 5.16$ & 0.043 & 0.225 & 0.043 \\
\hline Forearm /Antebraço & $23.64 \pm 5.90$ & $25.08 \pm 5.18$ & $25.58 \pm 5.52$ & 0.245 & 0.501 & 0.021 \\
\hline Leg / Perna & $28.80 \pm 5.60$ & $38.22 \pm 8.24$ & $30.50 \pm 3.10$ & 0.032 & 0.056 & 0.242 \\
\hline Group / Grupo 2 n = & & & & & & \\
\hline Forehead / Fronte & $27.95 \pm 3.92$ & $29.18 \pm 4.57$ & $33.45 \pm 7.97$ & 0.230 & 0.116 & 0.073 \\
\hline Zygomatic/Zigomática & $27.45 \pm 4.42$ & $30.53 \pm 5.18$ & $33.22 \pm 9.19$ & 0.173 & 0.500 & 0.075 \\
\hline Hand/ Mão & $34.67 \pm 5.31$ & $35.20 \pm 3.61$ & $32.48 \pm 5.41$ & 0.753 & 0.917 & 0.753 \\
\hline Forearm/Antebraço & $22.15 \pm 2.82$ & $23.30 \pm 2.28$ & $22.65 \pm 2.27$ & 0.223 & 0.450 & 0.746 \\
\hline Leg/ Perna & $24.80 \pm 5.24$ & $32.03 \pm 7.72$ & $34.25 \pm 6.40$ & 0.104 & 0.451 & 0.010 \\
\hline
\end{tabular}

During the study, TEWL was statistically significant in the hand in group 1 (Table 6) and in the leg in group 2 (Table 6), suggesting a slight increase in these more exposed areas where dryness is more obvious.

Table 6 - Transepidermal water Loss (g/h.m2)

Tabela 6 -Perda Transepidérmica de água (g/h.m2)
No decorrer do estudo a média dos valores da perda trans-epidérmica de água (PTEA) foi estatisticamente significativa na zona anatómica da mão no grupo 1 (tabela 6) e na zona anatómica da perna no grupo 2 (tabela 6), o que sugere um ligeiro aumento nas zonas anatómicas mais exposta que habitualmente apresentam a pele mais seca.

\begin{tabular}{|c|c|c|c|c|c|c|}
\hline & T0 & $\mathrm{T} 1$ & $\mathrm{~T} 2$ & $\begin{array}{l}\text { P-Value } \\
\text { (T1 vsT0) }\end{array}$ & $\begin{array}{l}\text { P-Value } \\
\text { (T2 vsT1) }\end{array}$ & $\begin{array}{c}\text { P-Value } \\
\text { (T2 vsT0) }\end{array}$ \\
\hline \multicolumn{7}{|l|}{ Group / Grupo 1 n $=5$} \\
\hline Forehead / Fronte & $9.06 \pm 2.34$ & $8.61 \pm 1.79$ & $8.68 \pm 2.99$ & 0.793 & 0.948 & 0.837 \\
\hline Zygomatic/Zigomática & $7.40 \pm 1.13$ & $8.74 \pm 0.95$ & $7.58 \pm 2.66$ & 0.093 & 0.336 & 0.859 \\
\hline Hand / Mão & $5.57 \pm 0.69$ & $6.61 \pm 0.70$ & $6.65 \pm 1.97$ & 0.043 & 0.498 & 0.500 \\
\hline Forearm /Antebraço & $5.02 \pm 1.00$ & $5.07 \pm 1.79$ & $4.44 \pm 0.81$ & 0.911 & 0.597 & 0.459 \\
\hline Leg / Perna & $4.73 \pm 1.35$ & $5.69 \pm 0.92$ & $7.18 \pm 3.22$ & 0.138 & 0.345 & 0.138 \\
\hline \multicolumn{7}{|l|}{ Group / Grupo 2 n $=6$} \\
\hline Forehead / Fronte & $11.85 \pm 3.63$ & $10.03 \pm 4.29$ & $9.36 \pm 2.11$ & 0.280 & 0.619 & 0.167 \\
\hline Zygomatic/Zigomática & $9.27 \pm 3.20$ & $10.32 \pm 5.87$ & $9.08 \pm 1.81$ & 0.659 & 0.544 & 0.888 \\
\hline Hand/ Mão & $6.92 \pm 1.57$ & $7.72 \pm 1.82$ & $8.68 \pm 4.65$ & 0.116 & 0.600 & 0.600 \\
\hline Forearm/Antebraço & $5.41 \pm 1.58$ & $5.78 \pm 1.69$ & $4.78 \pm 0.83$ & 0.478 & 0.182 & 0.169 \\
\hline Leg/ Perna & $4.94 \pm 2.29$ & $5.98 \pm 1.96$ & $5.33 \pm, 088$ & 0.046 & 0.345 & 0.463 \\
\hline
\end{tabular}




\section{Discussion}

The results of this study demonstrate that the additional water intake of (2L/daily) does not involve any increase in weight or volume change in the body water distribution, meaning that these skin physiology modifications may be directly related to the experimented changes to water in the diet.

The positive impact on skin hydration, in particular in the group of individuals whose total water intake was less than 2L/day, suggested greater (skin moisturizing) benefits. These are similar to those obtained in previous studies which evaluated the effect of an increased dietary intake of water on dry skin ${ }^{[7] 7}$. The magnitude of these changes is also smaller in the zygomatic area. This is usually an area where cosmetics are regularly applied. This suggests that hydration changes may be more noticeable in less hydrated areas.

In those areas where significant changes can already be observed in $\mathrm{T} 1$, the increase in hydration progresses with lower magnitude until the end of the study (T2) (Table 4). This may simply reflect the local epidermal water dynamics related to the skin water content. Drier areas initally tend to retain higher amounts of water, which necessarily reduces the magnitude of water retention along the course of time.

In relation to the impact on deep hydration, results have shown that changes in superficial hydration clearly modify deep skin hydration. This improves all skin functions ${ }^{[6]}{ }^{6}$ because more water is available for normal physiological processes ${ }^{[4,5] 4.5}$.

The statistically significant changes observed in the TEWL suggest, as previously published ${ }^{[5]}{ }^{5}$, that the increased dietary intake of water has little impact on barrier function. However it is possible to observe statistically significant changes in those locations where higher water increment occurs, as observed in the hand and the external part of the leg.

\section{Conclusions}

Although preliminary in nature, the results obtained in this study constitute the first evidence of a direct relationship between the water consumed in the diet and skin hydration. The topic still raises many questions that must necessarily involve more individuals in order to assess the impact of the water consumed in the daily diet on the normal physiology of the skin.

\section{Discussão}

Os resultados deste estudo demonstram que o aporte adicional de água (2L/dia) não envolve qualquer aumento ponderal nem alteração do volume de distribuição da água no organismo, o que significa que os resultados detectados sobre a fisiologia cutânea podem ser directamente relacionados com as alterações dietárias de água introduzidas.

O impacto positivo no aumento da hidratação cutânea em especial no grupo de indivíduos cujo aporte total de água é menor do que 2L/dia sugere benefícios em termos da hidratação da pele humana semelhantes aos obtidos em estudos anteriores que avaliaram o aumento do aporte dietários de água em pele seca ${ }^{7}$. A magnitude de alteração é igualmente menor na zona zigomática, área onde a aplicação de hidratantes é usual, confirmando que as alterações na hidratação podem ser maiores nas zonas que menos hidratadas.

Nas zonas anatómicas em que as alterações observadas são significativas em T1, o aumento da hidratação é de menor magnitude até final do estudo (T2) (tabela 4). Isto pode apenas refletir a dinâmica de água cutânea nesses locais, já que as áreas mais "sêcas" serão aquelas que, num primeiro momento, reterão mais àgu. Essa dinâmica de retenção, irá então diminuir ao longo do tempo.

Sobre o impacto na hidratação profunda, os resultados obtidos evidenciam que as alterações na hidratação superficial têm repercussões na hidratação cutânea profunda, o que sugere uma melhoria nas funções da pele ${ }^{6}$ pelo aumento do teor em água na pele disponível para os processos fisiológicos normais ${ }^{4,5}$.

As alterações estatisticamente significativas observadas na PTEA sugerem, à semelhança de estudos anteriores $^{5}$, que o aumento do aporte dietário de água tem pouco impacto na função barreira, sendo contudo possível observar alterações estatisticamente significativas nos locais em que o incremento do teor em água é maior, como acontece na mão e na face externa da perna.

\section{Conclusões}

Apesar do seu carácter preliminar, o conjunto de resultados aqui reunidos constitui a primeira evidência de uma relação directa, entre a água consumida na dieta e o reforço da hidratação cutânea. O tema encerra ainda muitas perguntas que, necessariamente, devem envolver mais indivíduos de forma a avaliar o impacto da água consumida na dieta quotidiana sobre a normal fisiologia da pele. 


\section{Acknowledgements}

To Carla Maria Lopes de Moura, Professor at the University of Porto, and to all volunteers who acceded to participate and collaborate in the data collection.

\section{Conflict of interest}

The authors declare that there is no financial or personal relationship that might be perceived as posing a potential conflict of interest.

\section{Agradecimentos}

A Carla Maria de Moura Lopes, Professora da Universidade do Porto, e a todas as voluntárias que acederam em participar e colaborar na recolha de dados.

\section{Conflito de Interesses}

Os autores declaram não existir qualquer relação pessoal ou financeira que possa ser entendida como representando um potencial conflito de interesses.

\section{References / Referências}

[1]. Nicolaidis S. Physiology and thirst. In: Hydration Throughout life, John Libbey Eurotext. 1998, p. 247.

[2]. Palma M: Characterization of the pattern of water consumption of a healthy population. [Master Thesis] Lisbon: ULHT 2012, page 11.

[3].Verdier-Sévrain S, Bonté F. Skin hydration: a review on its molecular mechanisms. Journal of Cosmetic Dermatology, 2007; 6:75-82.

[4]. Leyden J, Rawlings A. Skin moisturization, Marcel Dekker, Inc, New York, Basel Cosmetic Science and Technology Series, Vol 25, 2002: 304
[5]. Mary S, Creidi P, at al Assessment of effects of an additional dietary natural mineral water uptake on skin hydration in healthy subjects by dynamic barrier function measuremente and scoring clinic. Skin Research and Technology, 2006, 12: 199-205. [6].http://www.wma.net/en/30publications/10polici es/b3/ (08/11/2012)

[7].Willett W: Nutritional Epidemiology New York: Oxford University Press, 1998

[8].Palma M, Monteiro C, Bujan M, Rodrigues Monteiro. Water intake and it's relationship with the water content of the diet in a healthy population.
Biopharmaceuthical and Biomedical Research 2011 (8) :261-271.

[9].Krause's. Food, Nutrition and Diet Therapy, 12th ed. 2008 , pag146

[10.]http://www.efsa.europa.eu/en/efsajournal/pub/ 1459.htm $(2012 / 11 / 08)$

[11]. http://www.min saude.pt/ portal/ conteudos/ enciclopedia $+\mathrm{da}+$ saude/obesidade/comosediagnost icaaobesidade.htm (2012/11/08).

[12].Grimmes S, Martinsen OG. Bioimpedance and bioelectricity basics. 1st ed. London: Academic Press, 2000. 